msh-mss Mathématiques et sciences humaines

194 | Eté 2011

Varia

\title{
Thierry Martin (sous la dir.), Les sciences humaines sont-elles des sciences?
}

Paris, Vuibert, 2011

\section{Camille Roth}

\section{OpenEdition}

\section{Journals}

Édition électronique

URL : http://journals.openedition.org/msh/12045

DOI : $10.4000 / \mathrm{msh} .12045$

ISSN : 1950-6821

\section{Éditeur}

Centre d'analyse et de mathématique sociales de l'EHESS

Édition imprimée

Date de publication : 1 septembre 2011

Pagination : 71-72

ISSN : 0987-6936

\section{Référence électronique}

Camille Roth, "Thierry Martin (sous la dir.), Les sciences humaines sont-elles des sciences? ?,

Mathématiques et sciences humaines [En ligne], 194 | Eté 2011, mis en ligne le 02 septembre 2011, consulté le 21 septembre 2020. URL : http://journals.openedition.org/msh/12045 ; DOI : https:// doi.org/10.4000/msh.12045 


\section{ANALYSE BIBLIOGRAPHIQUE}

Thierry Martin (sous la dir.), Les sciences humaines sont-elles des sciences?, Paris, Vuibert, 2011.

À partir d'une question-titre relativement classique, l'ouvrage coordonné par T. Martin pourrait procéder d'une approche dialectique séparant tenants de la spécificité inaliénable et constitutive des sciences humaines, et avocats de leur digne scientificité. L'ouvrage parvient avec succès à éviter cette polarisation, pour mieux faire ressortir les caractéristiques partagées à la fois par les sciences naturelles et humaines. Les deux premiers chapitres mettent en place clairement et immédiatement les différents axes de ce rapprochement. Premier axe, D. Andler rappelle les enjeux des programmes naturaliste et réductionniste en sciences humaines et suggère en particulier que leur succès n'est pas une condition nécessaire à la scientificité des sciences humaines. Sa contribution dessine notamment une divergence entre d'une part le pari de naturalisation des sciences sans exception et d'autre part l'ambition plus modeste d'introduire des approches formelles et quantitatives au sein de ces disciplines, ambition qui permet déjà d'assurer leur scientificité sans forcément compromettre les particularités ontologiques de chacune d'elles.

A. Bouvier esquisse le deuxième axe en rappelant pertinemment que toutes les disciplines, en sciences naturelles comme humaines, sont traversées par l'opposition entre approches «idiographiques » (liées à la description de phénomènes relativement singuliers) et approches "nomologiques» (liées à la découverte de lois ou de mécanismes relativement universels). Malgré ses racines anciennes, ce point de vue peut même apparaître comme le plus audacieux de l'ouvrage; son argumentation solide parvient à questionner efficacement aussi bien la spécificité des sciences humaines que celle des sciences naturelles.

Les deux contributions suivantes fournissent des illustrations disciplinaires plus concrètes. Le chapitre de D. Raynaud montre bien en quoi une portion des résultats contemporains de la physique statistique est pertinente pour étudier les phénomènes et processus sociologiques, notamment dans leurs aspects les plus nomologiques. La démonstration pourrait ici toutefois être renforcée par l'exemple plus ancien de l'analyse des réseaux sociaux comme champ de recherche employant des "méthodes scientifiques ordinaires » en sociologie et, en tant que tel, pouvant déjà être qualifié de science normale - car, dans ce contexte, les sciences naturelles semblent avoir émergé au sein même de la sociologie.

L. Sanders adopte ensuite une approche davantage historique qu'épistémologique en décrivant la manière dont les méthodes formelles et quantitatives - jusqu'aux modèles multi-agents - ont progressivement été introduites en géographie au cours des dernières décennies, et comment les courants porteurs de ces changements se sont structurés et ont assis leur légitimité. Ces études de cas issues de domaines distincts couvrent bien deux des processus les plus importants à l'établissement de la scientificité d'une discipline : la justification de la pertinence épistémologique de ces nouvelles méthodes, d'abord, mais aussi l'opportunité pour les acteurs de mettre au point un paradigme leur permettant de se rassembler autour de ces outils.

Dans un dernier temps, l'ouvrage s'attache à discuter plus précisément le cas de l'intentionnalité comme point focal de la spécificité des sciences humaines. G. Lenclud propose d'abord une discussion détaillée de la particularité de l'explication historique et de son éloignement du modèle nomologique - son chapitre paraît à cet égard être celui 
qui défend probablement le plus une spécificité des sciences humaines: celles-ci restant ainsi prisonnières d'une incertitude minimale lors de l'opération d'attribution d'intentions aux individus, opération nécessaire à l'explication. Dans la continuité des parallèles tracés par les auteurs des chapitres précédents, le lecteur pourrait ici toutefois s'interroger sur l'existence de cas semblables en sciences naturelles - concernant par exemple la place du fonctionnalisme en biologie - et considérer en d'autres termes que d'autres disciplines rencontrent aussi cette forme de sous-détermination des théories par les faits. La contribution de D. Bonnay et M. Cozic offre alors un contrepoint bienvenu à l'idée que la tâche de compréhension des actions humaines reste irréductiblement au cœur de la spécificité des sciences humaines et, dans une certaine mesure, ne se prête pas à la naturalisation. En critiquant le principe de charité et, plus largement, en effectuant un exposé fourni et parfois véritablement technique des débats concernant les théories de la rationalité, ce chapitre revient ensuite au cœur du sujet en montrant que l'intentionnalité appréhendée sous l'angle d'une rationalité normative peut poser problème et est (ou devrait davantage) être un terrain de recherche propre. De fait, l'argument plaide davantage pour l'unité des sciences: dès lors que les chercheurs s'appuient sur un modèle comportemental pour expliquer les actions humaines et leurs motifs, les différences avec l'explication des phénomènes biologiques ou physiques semblent s'estomper.

Le manuscrit peut ainsi, plus généralement, renvoyer l'image d'un questionnement en forme de double affaiblissement des spécificités conventionnellement attribuées aux sciences humaines comme aux sciences dures; même s'il apparaît qu'il n'y a probablement pas consensus à ce sujet parmi les différents contributeurs. Quelle qu'en soit la lecture, cet ouvrage collectif offre finalement une contribution singulière et très stimulante à propos d'un débat traditionnel mais aussi très contemporain, du fait de l'essor massif des sciences cognitives d'une part, et des sciences sociales formelles/computationnelles d'autre part. En ce sens, il pourrait parfaitement avoir été écrit à l'attention spécifique de l'audience d'une revue comme Mathématiques et Sciences humaines, même s'il donne parfois l'impression de s'appuyer davantage sur des exemples inspirés des sciences cognitives plutôt que sociales. L'ouvrage peut en outre s'adresser, étonnamment, à un public plus averti qu'il n'y paraît : un public à la fois bien informé sur les débats épistémologiques concernant le statut scientifique des sciences humaines et, de manière plus inattendue, familier de l'expérience du praticien des sciences naturelles en sciences humaines.

C. Roth 